\title{
Efeitos de isolados do fungo Isaria (Persoon) sobre o cupim subterrâneo Coptotermes gestroi (Wasmann) (Isoptera: Rhinotermitidae)
}

\author{
Effects of Isaria (Persoon) isolates on the subterranean termite Coptotermes \\ gestroi (Wasmann) (Isoptera: Rhinotermitidae)
}

\author{
Eliana Maria dos Passos ${ }^{1 *}$, Auristela Correia Albuquerque ${ }^{2}$, Edmilson Jacinto Marques ${ }^{1}$, \\ Valéria Wanderley Teixeira ${ }^{3}$, Cinthia Conceição Matias da Silva ${ }^{1}$, Marco Aurélio Paes de Oliveira ${ }^{2}$

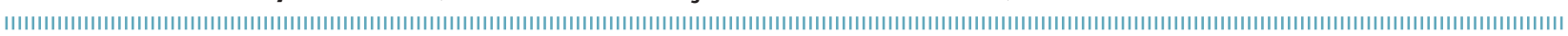

RESUMO: Coptotermes gestroi (Wasmann) é considerada uma das espécies mais destrutivas de cupins subterrâneos, por causar danos a edificaçóes, à arborização urbana e a culturas. Uma alternativa para o controle desse inseto pode ser o uso de agentes biocontroladores, como os fungos entomopatogênicos. Isaria (Persoon) tem sido indicado no controle de térmitas subterrâneos, inclusive do gênero Coptotermes. Dessa forma, o presente trabalho teve como objetivo selecionar isolados de Isaria patogênicos ao cupim C. gestroi. Os insetos pulverizados com suspensões fúngicas de I. farinosa, I. fumosorosea e I. javanica foram avaliados diariamente para a determinaçáo da mortalidade. Todos os isolados foram patogênicos, ocasionando mortalidade acima de $70 \%$, e virulentos, apresentando uma sobrevivência média de 2,0 a 3,9 dias. Contudo, os isolados ESALQ-1205 de I. farinosa, ESALQ-1296 de I. fumosorosea e os isolados URM-4995 e URM-4993 de I. javanica mostraram-se mais virulentos. $\mathrm{A} \mathrm{CL}_{50}$ estimada para os isolados ESALQ-1205 de I. farinosa, URM-4995 de I. javanica e ESALQ-1296 de I. fumosorosea resultou em valores de 3,7 x 105, $1,4 \times 10^{6}$ e 2,7 x $10^{6}$ conídios $\mathrm{mL}^{-1}$, respectivamente. Tais resultados confirmam a eficiência dos isolados testados sobre os operários de C. gestroi. No entanto, novos estudos são necessários para verificar a melhor forma de utilizaçáo, bem como a sua efetividade em campo.

PALAVRAS-CHAVE: térmitas; controle microbiano; patogenicidade; sobrevivência.
ABSTRACT: Coptotermes gestroi (Wasmann) is considered to be one of the most destructive species of subterranean termites because of the damage caused on edifications, urban trees, and crops. One alternative to control it is the use of biocontrol agents, such as the entomopathogenic fungi. The genus Isaria (Persoon) has been suggested for the control of subterranean termites, including the Coptotermes genus. Therefore, the present work aim was to select isolates of Isaria pathogenic to $C$. gestroi. The workers were sprayed with fungi suspensions of I. farinosa, I. fumosorosea and I. javanica, and evaluated every day to determine mortality. All isolates were pathogenic towards C. gestroi with mortality rates higher than $70 \%$, and virulent, with mean of survival of 2.0 up to 3.9 days. Among the isolates, the ESALQ-1205 isolate of I. farinosa, the ESALQ-1296 isolate of I. fumosorosea, URM-4995 and URM-4993 isolates of I. javanica showed the highest virulence. The estimated $\mathrm{CL}_{50}$ for the ESALQ-1205 isolate of $I$. farinosa, URM-4995 isolate of $I$. javanica and ESALQ-1296 isolate of $I$. fumosorosea was $3.7 \times 10^{5}, 1.4 \times 10^{6}$ and $2.7 \times 10^{6}$ conidia $\mathrm{mL}^{-1}$, respectively. These results suggest high efficiency of these isolates towards the C. gestroi workers. Nevertheless, further studies are necessary to determine a way of using them as well as their efficacy in field.

KEYWORDS: termites; microbial control; pathogenicity; survivorship. 


\section{INTRODUÇÃO}

Coptotermes gestroi (WASMANN) é um térmita subterrâneo de origem asiática, considerado como praga agrícola e de edificações, o qual vem se estabelecendo nas regiôes tropical e subtropical do mundo (Jenkins et al., 2007). No Brasil, existem grandes infestaçôes na região Sudeste, além de ocorrências registradas nos estados de Pernambuco (Fontes; Veiga, 1998), Ceará, Bahia, Pará e Paraná (Ferraz, 2000). Os indivíduos desta espécie, além de causarem enormes danos a edificaçóes urbanas ou rurais, comprometem a arborizaçấo urbana e são considerados como praga agrícola (Fontes, 1995; Costa-Leonardo, 2002).

A principal tática de controle utilizada para diminuir as infestaçôes de térmitas subterrâneos como o C. gestroi é a aplicaçáo de inseticidas. No entanto, mesmo com a substituição dos clorados por outros grupos de inseticidas de menor poder residual, como piretroides, neonicotinoides, fenil pirazol, reguladores de crescimento e éter difenílico, a utilização desses produtos ainda apresenta alguma limitação, como o efeito residual (Рotenza; Zorzenon, 2006). O controle de C. gestroi se torna ainda mais difícil devido ao enorme tamanho de suas populaçóes, ninhos policálicos, presença de neotênicos e forrageamento a longas distâncias (Costa-Leonardo, 2002).

Uma alternativa para o controle desse inseto pode ser o uso de agentes biocontroladores, especialmente os fungos entomopatogênicos, pois as condiçôes dos ninhos dos cupins, com temperatura moderada e umidade alta, contribuem para o crescimento de espécies de fungos e são fatores importantes para a sobrevivência e a propagação desses agentes de controle biológico (Kramm et al., 1982; Ignoffo, 1992). Chouvenc et al. (2011), analisando 50 anos de pesquisa sobre o controle microbiano de térmitas, constataram que $65 \%$ de todos os experimentos relatados testaram uma espécie fúngica; das mais de 20 espécies testadas para a patogenicidade, cerca de 15 mostraram-se virulentas.

Estudos têm demonstrado que Metarhizium anisopliae (Mestsch.) Sorok. é virulento a térmitas subterrâneos e causa grande mortalidade em alados e operários de Coptotermes (Wright et al., 2005; Maketon et al., 2007). Além disso, não apresentam repelência a esses térmitas, sendo bons candidatos para o uso em campo (Wright; Cornelius, 2012). Sugere-se ainda seu uso como isca, em uma mistura de conídios e atrativos (Wang; Powell, 2004; Balachander et al., 2013).

Contudo, fungos do gênero Isaria (Persoon) têm se desatacado no controle de espécies de térmitas subterrâneas, através da utilização de linhagens de Isaria fumesorosea (=Paecilomyces fumosoroseus) (Wize) e Isaria javanica (=Paecilomyces javanicus) (Friedrichs e Bally), que produzem grande quantidade de inóculo em meios sólidos. São fáceis e baratos de serem preparados, além de não serem repelentes, o que facilita a disseminação entre os indivíduos (Wright et al., 2003; MeikLE et al., 2005; Wright et al., 2008; YANAGAWA et al., 2008; WRIGHT;
Cornelius, 2012; Dunlap et al., 2012). O uso deste patógeno tem sido pesquisado e recomendado para o controle desses térmitas na forma de pó seco, pó molhável associado ou não com queratina hidrolisada (WRIGHT et al., 2003; Dunlap et al., 2007; Wright et al., 2008, 2012). A espuma de queratina foi desenvolvida como um mecanismo de liberação biologicamente compatível com o agente de controle microbiano, para permitir a expansão do patógeno no cupinzeiro (Dunlap et al., 2007, 2012).

Além disso, Isaria spp. é empregado em escala comercial em cultivos protegidos na Europa e nas Américas do Norte e Latina, para o controle de pulgôes, mosca-branca, tripes, cochonilhas, ácaros, coleópteros e cigarrinhas (FARIA; MAGAlHães, 2001; Alves et al., 2008). No Brasil, Isaria spp. tem sido produzido no estado de Mato Grosso para o controle do percevejo de renda da seringueira, Leptopharsa heveae (Drake \& Poor) (Heteroptera: Tingidae) (Alves et al., 2008).

Tendo em vista que em programas de controle microbiano a seleção de isolados patogênicos para o inseto alvo é uma etapa de fundamental importância, o trabalho teve como objetivo verificar o efeito dos isolados de três espécies de Isaria sobre o cupim C. gestroi.

\section{MATERIAL E MÉTODOS}

\section{Obtenção dos insetos}

Os insetos foram coletados mediante o uso de armadilhas de papeláo corrugado, envoltas em tubo plástico de acordo com a metodologia descrita por Costa-Leonardo (2002). As armadilhas foram colocadas diretamente no solo, próximas a um tronco de abacateiro (Persea americana Mill) infestado de C. gestroi, localizado no bairro de Iputinga, Recife (PE). Posteriormente, as iscas com os cupins foram acondicionadas em laboratório, com temperatura aproximada de $26 \pm 2^{\circ} \mathrm{C}$, umidade acima de $60 \%$ e escotofase constante.

\section{Obtenção, multiplicação e viabilidade dos isolados}

Os isolados investigados de Isaria obtidos de diferentes hospedeiros e localidades (Tabela 1) foram mantidos na micoteca do Laboratório de Patologia de Insetos da UFRPE, após revigoramento em operários de C. gestroi.

Inicialmente, os isolados foram repicados para placas de Petri, contendo meio batata-dextrose-ágar mais antibiótico $(\mathrm{BDA}+\mathrm{A})$. Após sete dias foram feitas placas cheias contendo meio completo (MC), constituído de extrato de levedura, glucose, sais minerais, ágar e água destilada, sendo os isolados uniformemente espalhados por toda a extensão da placa, com o auxílio da alça de Drigalsky. As placas permaneceram 
Tabela 1. Procedência (localidade e hospedeiro) dos isolados de Isaria farinosa, Isaria javanica e Isaria fumosorosea utilizados sobre Coptotermes gestroi.

\begin{tabular}{ccc} 
Isolados & \multicolumn{1}{c}{ Localidade } & Hospedeiros \\
I. farinosa & & \\
\hline ESALQ-1205 & Santa Fé do Sul- SP & Bemisia tabaci \\
\hline ESALQ-1355 & Aldeia- PE & Brassolis sopharae \\
\hline I. javanica & & \\
\hline URM-4993 & Rio Grande do Sul & Coleóptero \\
\hline URM- 4995 & Rio Grande do Sul & Coleóptero \\
\hline I. fumosorosea & & \\
\hline ESALQ-1296 & Piracicaba- SP & B. tabaci \\
\hline ESALQ-1297 & Ipeúna- SP & Lagria villosa \\
\hline
\end{tabular}

em estufa incubadora B.O.D. a $26 \pm 1^{\circ} \mathrm{C}$, com fotofase de 12 horas para germinação e crescimento dos isolados.

Posteriormente, foram preparadas suspensôes fúngicas, adicionando-se $10 \mathrm{~mL}$ de água destilada esterilizada mais espalhante adesivo Tween 80 (0,01\%), sendo os conídios removidos com a ajuda de uma espátula de borracha. As suspensóes resultantes, depois de filtradas em gaze esterilizada, foram aferidas e ajustadas para concentração de $10^{7}$ conídios $\mathrm{mL}^{-1}$ mediante quantificação em câmara de Neubauer, utilizando-se microscópio óptico.

A viabilidade dos isolados foi avaliada segundo a metodologia descrita por Alves; Moraes (1998).

\section{Verificação da patogenicidade e virulência dos isolados}

$\mathrm{Na}$ avaliação da patogenicidade, para cada isolado, 70 operários de C. gestroi agrupados em uma placa de Petri $(15 \times 2,0 \mathrm{~cm})$ foram pulverizados de maneira uniforme e simultânea, com $1 \mathrm{~mL}$ de suspensão a $10^{7}$ conídios $\mathrm{mL}^{-1}$, utilizando-se microatomizador "Paasche Airbrush" elétrico, modelo "VL", acoplado a um compressor regulado para 5 libras de pressão. $\mathrm{Na}$ testemunha, os insetos foram pulverizados com água destilada e espalhante adesivo a 0,01\%. Após a aplicação, com o auxílio de um pincel umedecido, os cupins foram transferidos em grupos de 10 para placas de Petri $(9,0 \mathrm{~cm})$ forradas com papel de filtro umedecido, contendo pedaços de papeláo corrugado $(2,0 \times 2,0 \mathrm{~cm})$, o qual servia como abrigo e fonte de alimento. Para a manutenção da umidade, as placas com os cupins eram fechadas com filme plástico. Os cupins tratados foram mantidos em temperatura ambiente $26 \pm 2{ }^{\circ} \mathrm{C}$ e escotofase constante. $\mathrm{O}$ experimento foi conduzido em delineamento inteiramente casualizado, com sete tratamentos e sete repetiçóes, sendo cada parcela constituída por 10 operários.
A mortalidade foi avaliada diariamente, e os cupins mortos foram transferidos para câmara úmida, mantidos em B.O.D. a $26 \pm 1^{\circ} \mathrm{C}$ e 12 horas de fotofase para confirmação do agente causal. Os dados de mortalidade média foram transformados em arco-seno $\sqrt{ }(x+0,5)$ e submetidos à análise de variância (ANOVA), utilizando o Proc ANOVA do SAS (SAS InSTITUTE, 1999-2001); as médias foram comparadas pelo teste de Tukey a $5 \%$ de probabilidade.

Mediante os dados de mortalidade confirmada, determinou-se a porcentagem de sobrevivência média. Os dados foram submetidos ao teste Long-Rank, através do método KaplanMeyer por pares de isolados, usando o Proc Lifetest do SAS (SAS Institute, 1999-2001).

\section{Estimativa da $\mathrm{CL}_{50}$}

A partir dos resultados obtidos no teste de patogenicidade, um isolado de cada espécie foi selecionado para investigação da concentração letal. Suspensóes dos isolados ESALQ-1205 de Isaria farinosa (=Paecilomyces farinosus) (Holmsk.) Fr., ESALQ-1296 de I. fumosorosea e URM-4995 de I. javanica foram ajustadas a $10^{5}, 5 \times 10^{5}, 10^{6}, 5 \times 10^{6}, 10^{7}, 5 \times 10^{7}, 10^{8}$ conídios $\mathrm{mL}^{-1}$ e usadas para pulverizar os cupins, compondo os tratamentos. No grupo controle foi aplicada água destilada esterilizada e Tween (80) a 0,01\%. Para cada tratamento foram utilizadas seis repetiçóes com 12 operários pulverizados, com $1 \mathrm{~mL}$ de cada suspensão. Na preparação e aplicação das suspensóes, determinação da viabilidade de conídios, acomodação dos cupins e avaliaçóes, foi utilizada a mesma metodologia empregada no experimento de patogenicidade. A mortalidade foi avaliada por um período de quatro dias, e os insetos mortos foram transferidos para a câmara úmida, sendo mantidos em B.O.D. a $26 \pm 1^{\circ} \mathrm{C}$ e 12 horas de fotofase para confirmação do agente causal. A partir dos dados de mortalidade confirmada estimou-se a $\mathrm{CL}_{50}$, empregando-se o Proc Probit do programa estatístico SAS (SAS InSTITUTe, 1999-2001).

\section{RESULTADOS E DISCUSSÃO}

\section{Patogenicidade e virulência dos isolados}

Os isolados de Isaria spp. testados apresentaram viabilidade superior a 95\%, o que demonstra a alta capacidade germinativa dos mesmos. As mortalidades obtidas através dos isolados de I. farinosa, I. javanica e I. fumosorosea foram superiores a $70 \%$, enquanto na testemunha esta porcentagem foi inferior a 10\%, não sendo necessário corrigir a mortalidade, demonstrando que todos os isolados são patogênicos ao cupim C. gestroi, capazes de desencadear doença e levar à morte. Contudo, os valores de mortalidade confirmada diferiram significativamente 
entre o isolado ESALQ-1297 de I. fumosorosea, que ocasionou 70,24\% de mortalidade, e os isolados ESALQ-1205 de I. farinosa e URM-4995 de I. javanica, que causaram mortalidades de 97,14 e $100 \%$, respectivamente (Tabela 2).

A sobrevivência, ou seja, o tempo de vida dos cupins após receberem o tratamento com as suspensões fúngicas, nas condiçôes de laboratório previamente relatadas, foi demonstrada em média (Tabela 2) e em sobrevivência diária (Fig. 1). Ambas indicam a intensidade ou o grau com que o patógeno causa a enfermidade. Com o tratamento fúngico, os cupins apresentaram uma sobrevivência média de 2,0 a 3,9 dias, diferindo significativamente entre os isolados, e destes com o grupo controle, que apresentou valor médio de sobrevivência de 13,7 dias (Tabela 2). Os isolados se comportaram como um patógeno rápido sobre o hospedeiro, e, portanto também são adequados como inseticidas microbianos em introdução inundativa (Alves; LeuConA, 1998).

Diferenças na sobrevivência diária entre o grupo controle e os tratamentos reforçam o efeito patogênico dos isolados sobre o cupim C. gestroi. Observou-se tempo de vida de até 35 dias para os cupins pertencentes ao grupo controle, e de no máximo 7 dias nos tratamentos (Fig. 1). Já a variação na sobrevivência entre os isolados indica diferentes níveis da doença ocasionada por eles, sendo que os isolados ESALQ-1297 de I. fumosorosea e ESALQ-1355 de I. farinosa sobrepôem-se e são responsáveis por maior tempo de vida em relação aos demais isolados, demonstrando menor agilidade no processo de infecção (Fig. 1).

Assim, de acordo com os dados de mortalidade e sobrevivência apresentados, pode-se afirmar que os isolados ESALQ-1205 de I. farinosa, ESALQ-1296 de I. fumosorosea e os isolados URM-4995 e URM-4993 de I. javanica mostraram-se mais virulentos sobre o cupim C. gestroi, ocasionando percentuais elevados de mortalidade em menor período de tempo.

Embora os isolados de I. javanica tenham se destacado entre os mais virulentos, o estudo de aspectos morfológicos desses dois isolados, também sobre C. gestroi, constatou maior crescimento da colônia de URM-4995 (Lopes et al., 2011), confirmando a sua indicação, visto que tal característica favorece a disseminaçáo entre os cupins.

Quanto ao I. fumesorosea, este tem demonstrado ser eficiente sobre outras espécies de Coptotermes, chegando a ocasionar $100 \%$ de mortalidade sobre Coptotermes formosanus (Shiraki) (Yanagawa et al., 2008; Meikle et al., 2005). Testes avaliando a sobrevivência de $C$. formosanus pulverizados com I. fumesorosea comprovaram que este é até mais virulento do que o produto comercial à base do fungo M. anisopliae (MeikLe et al., 2005), e ocasiona maior mortalidade aos térmitas do que este patógeno (Wright; Cornelius, 2012). Uma formulação à base de blastosporos de I. fumesorosea, com uso de queratina hidrolisada como surfactante, também já foi testada, patenteada e recomendada para o controle de C. formosanus (Dunlap et al., 2007, 2012). Estudos de comportamento
Tabela 2. Mortalidade confirmada (\%) aos cinco dias e sobrevivência média (dias) de Coptotermes gestroi por isolados de Isaria spp. $\left(10^{7}\right.$ conídios $\left.\mathrm{mL}^{-1}\right)$. Temp.: $26 \pm 2{ }^{\circ} \mathrm{C}$; UR $70 \pm 10 \%$ e escotofase.

\begin{tabular}{lcc} 
Isolados & Mortalidade (\%) & Sobrevivência (dias) \\
\hline ESALQ-1205 & $97,1 \pm 1,84 \mathrm{a}$ & $2,8 \pm 0,09 \mathrm{c}$ \\
\hline ESALQ-1297 & $70,24 \pm 9,90 \mathrm{~b}$ & $3,9 \pm 0,18 \mathrm{~b}$ \\
\hline ESALQ-1355 & $92,2 \pm 3,10 \mathrm{ab}$ & $3,7 \pm 0,15 \mathrm{~b}$ \\
\hline ESALQ-1296 & $92,5 \pm 3,8 \mathrm{ab}$ & $3,0 \pm 0,10 \mathrm{c}$ \\
\hline URM-4993 & $82,06 \pm 6,81 \mathrm{ab}$ & $2,8 \pm 0,12 \mathrm{c}$ \\
\hline URM-4995 & $100,0 \mathrm{a}$ & $2,0 \pm 0,09 \mathrm{c}$ \\
\hline Testemunha & - & $13,7 \pm 0,80 \mathrm{a}$ \\
\hline & $\mathrm{F}_{5 ; 41}=3,79^{\circ, 0074}$ & $\chi^{2}=246,3^{0,0001}$ \\
\hline
\end{tabular}

'Médias ( \pm EP) seguidas da mesma letra, na coluna, não diferem entre si pelo teste de Tukey; ${ }^{2}$ médias seguidas da mesma letra não diferem entre si pelo teste de Log-Rank por pares de isolados após análise de sobrevivência pelo método Kaplan-Meyer.

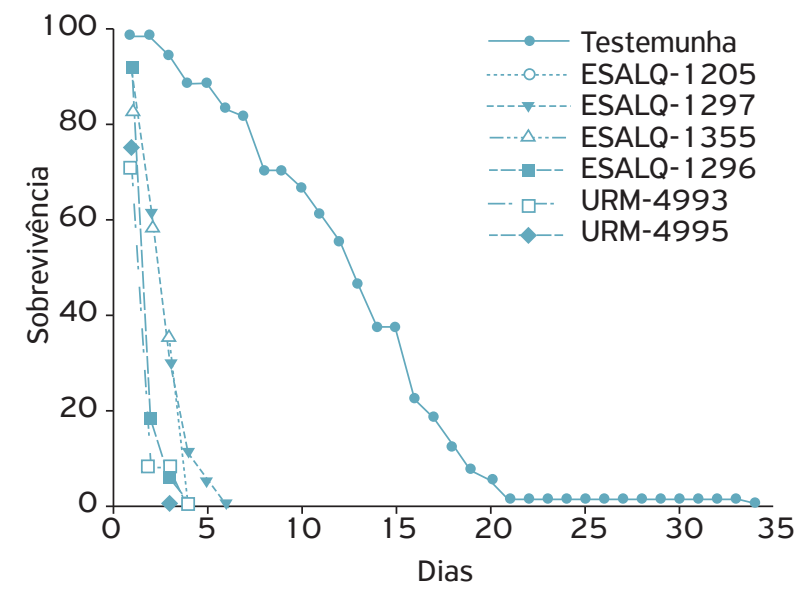

Figura 1. Sobrevivência diária (\%) do cupim Coptotermes gestroi tratado com isolados de Isaria spp. avaliada até a morte do ultimo indivíduo.

realizados recentemente mostraram ainda que este fungo náo é repelente a operários desses térmitas, o que os tornam altamente suscetíveis à infecção (HussaIn et al., 2010; WRIGHT; Cornelius, 2012), sugerindo possibilidade de utilização na forma de isca.

Embora existam diversos estudos a respeito do potencial no controle de térmitas com I. fumesorosea e poucos estudos com I. javanica, micoinseticidas à base de linhagens dessas duas espécies já foram patenteadas nos Estados Unidos, para o controle de cupins subterrâneos (WRight et al., 2003, 2008). Contudo, estudos a respeito de I. farinosa para o controle de térmitas são raros, ou mesmo inexistentes. Desta 
Tabela 3. Estimativa da $\mathrm{Cl}_{50}$ para os isolados de Isaria spp. sobre o cupim Coptotermes gestroi.

\begin{tabular}{|c|c|c|c|c|c|c|}
\hline Isolado & $\mathbf{n}$ & GL & $\begin{array}{c}\left.\mathrm{CL}_{50} \text { (Conídios } \mathrm{mL}\right) \\
\text { IC95\% }\end{array}$ & $\begin{array}{c}\text { Inclinação da reta } \\
(\beta \pm E P)\end{array}$ & $\chi^{2}$ & Valor $\mathrm{p}$ \\
\hline ESALQ- 1205 & 447 & 5 & $\begin{array}{c}3,7 \times 10^{5} \\
\left(2,9 \times 10^{5}-4,5 \times 10^{5}\right)\end{array}$ & $2,31 \pm 0,26$ & 0,76 & 0,9797 \\
\hline URM-4995 & 391 & 4 & $\begin{array}{c}1,4 \times 10^{6} \\
\left(1,2 \times 10^{6}-1,8 \times 10^{6}\right)\end{array}$ & $2,55 \pm 0,24$ & 2,18 & 0,7034 \\
\hline ESALQ- 1296 & 409 & 4 & $\begin{array}{c}2,7 \times 10^{6} \\
\left(2,3 \times 10^{6}-3,4 \times 10^{6}\right)\end{array}$ & $2,24 \pm 0,19$ & 2,80 & 0,5911 \\
\hline
\end{tabular}

GL: Grau de Liberdade para teste de qui-quadrado $\left(\chi^{2}\right)$; significância a $5 \%$ de probabilidade; ( $\left.\beta \pm E P\right)$ : coeficiente angular da reta \pm erro padrão.

forma, acredita-se que este trabalho seja o primeiro, ou um dos poucos, a registrar o potencial desta espécie de Isaria no controle desses insetos.

Durante as avaliações diárias foi possível observar, em todos os tratamentos e na testemunha, um comportamento de ataque, caracterizado pela presença de operários de C. gestroi com a cabeça separada do corpo, ou somente a cabeça. Esse ataque faz parte de um conjunto de comportamentos de defesa contra esse tipo de patógeno, também registrado nesse mesmo gênero de cupim, tratado com Isaria (YANAGAWA et al., 2011). Demonstra a tentativa de remover o material recém-aplicado, já que os ataques se concentraram basicamente nas primeiras 24 horas.

\section{Estimativa da $\mathrm{CL}_{50}$}

Como os isolados das três espécies estudadas se destacaram na avaliação da virulência, um isolado de cada espécie foi selecionado para investigação da concentração letal. Estimou-se, portanto, a $\mathrm{CL}_{50}$ para os isolados ESALQ-1205 de I. farinosa, URM-4995 de I. javanica e ESALQ-1296 de I. fumosorosea, resultando em valores de 3,7 × 105, 1,4 x $10^{6}$ e 2,7 x $10^{6}$ conídios $\mathrm{mL}^{-1}$, respectivamente. Estes diferem entre si pelo intervalo de confiança, contudo, nas curvas de concentração de mortalidade não foi observada diferença em relação à inclinação das retas (Tabela 3). Tais resultados corroboram os dados de mortalidade já apresentados, indicando não haver diferença na mortalidade ocasionada pelos isolados. Assim, confirma a eficiência sobre os operários de C. gestroi e demonstra que pode ser usado em uma dosagem menor, tornando-se mais econômico no caso de uma possível aplicação em campo.

\section{CONCLUSÕES}

Os isolados de I. farinosa, I. javanica e I. fumesorosea se mostraram patogênicos e virulentos, tendo os isolados ESALQ-1205 de I. farinosa, ESALQ-1296 de I. fumosorosea e os isolados URM-4995 e URM-4993 de I. javanica se destacado como mais virulentos. Portanto, são mais indicados no controle do cupim C. gestroi. As diferenças encontradas na proporção de cupins mortos e tempo de sobrevivência, após tratamento, devem-se à grande variabilidade genética existente entre isolados de espécies diferentes ou da mesma espécie. Os testes de patogenicidade e a avaliação da virulência, através da análise de sobrevivência, são fundamentais na seleção dos isolados mais promissores. Desta forma, este trabalho representa uma importante contribuição para o controle biológico de C. gestroi com o uso de fungos do gênero Isaria. Porém, são necessários estudos para verificar a melhor forma de utilização e sua efetividade em campo.

\section{REFERÊNCIAS}

ALVES, S.B.; LEUCONA, R.E. Epizootiologia aplicada ao controle microbiano de insetos. In: (Ed.). Controle Microbiano de Insetos. Piracicaba: FEALQ. 1998. p.97-169.

ALVES, S.B.; MORAES, A.S. Quantificação de inóculo de patógenos de insetos. In: (Ed.). Controle Microbiano de Insetos. Piracicaba: FEALQ. 1998. p.97-169.

ALVES, S.B.; LOPES, R.B.; VIEIRA, A.S.; TAMAI, M.A. Fungos Entomopatogênicos usados no controle de pragas na América Latina. In: ALVES, S.B.; LOPES, R.B.(Eds.). Controle Microbiano de Pragas na América Latina: avanços e desafios. Piracicaba. FEALQ. 2008. p.69-1 10.
CHOUVENC, T.; NAN-YAO, S.; GRACE, J. KENNETH. Fifty years of attempted biological control of termites - Analysis of a failure. Biological Control, v.59, n.2, p.69-82, 2011.

COSTA-LEONARDO, A.M. Cupins-Praga: Morfologia, Biologia e Controle. Rio Claro: UNESP. 2002. 128 p.

DUNLAP, C.A.; JACKSON, M. A.; WRIGHT, M. S. A foam formulation of Paecilomyces fumosoroseus, an entomopathogenic biocontrol agent. Biocontrol Science Technology, v.17, n.9, p.709-719, 2007. 
DUNLAP, C.A.; JACKSON, M. A.; WRIGHT, M. S. Compositions of keratin hydrolysate and microbes for pest control applications. US Patent 8263526. 2012.

FARIA, M.R.; MAGALHÃES, B.P. O uso de fungos entomopatogênicos no Brasil. Biotecnologia Ciência e Desenvolvimento, n.22, p.8-44, 2001.

FERRAZ, M.V. Estudo taxonômico e aspectos da biologia de Coptotermes Wasmann, (Isoptera, Rhinotermitidae) nas Américas. 2000. 213 f. Tese (Doutorado) - Universidade de São Paulo, 2000.

FONTES, L.R. Cupins em áreas urbanas. In: BERTI FILHO, E.; FONTES, L.R.(Eds.). Alguns aspectos atuais da biologia e controle de cupins. Piracicaba: FEALQ. 1995. p.57-75.

FONTES, L.R., VEIGA, A.F.S.L. Registro do cupim subterrâneo, Coptotermes havilandi (Isoptera, Rhinotermitidae), na área metropolitana de Recife, PE. Anais da Sociedade Brasileira de Entomologia, Rio de Janeiro, 1005p, 1998.

HUSSAIN, A.; TIAN, M.Y.; HE, Y.R.; BLAND, J.M.; GU, W.X. Behavioral electrophysiological responses of Coptotermes formosanus Shiraki towards entomopathogenic fungal volatiles. Biological Control, v.55, n.3, p.166-173, 2010.

IGNOFFO, C.M. Environmental factors affecting persistence of entomopathogens. Florida Entomologist, v.75, n.4, p.516-525, 1992.

JENKINS, T.M.; JONES, S.C.; LEE, C.Y.; FORSCHLER, B.T.; CHEN, Z.; LOPEZ-MARTINEZ, G.; GALLAGHER, N.T.; BROWN, G.; NEAL, M.; THISTLETON, B.; KLEINSCHMIDT, S. Hylogeography illuminates maternal origins of exotic Coptotermes gestroi (Isoptera: Rhinotermitidae). Molecular Phylogenetics and Evolution, v.42, n.3, p.612-621, 2007.

KRAMM, K.R.; WEST, D.F.; ROCKENBACH, P.G. Termite pathogens: transfer of the entomopathogen Metarhizium anisopliae between Reticulitermes sp. Termites. Journal of Invertebrate Pathology, v.39, n. 1, p.1-5, 1982.
LOPES, R.S.; SVEDESE, V.M.; SILVA, A.P.A.P., ALBUQUERQUE, A.C.; LUNA-ALVES LIMA, E.A. Virulência e Aspectos biológicos de Isaria javanica (Frieder \& Bally) Samson \& Hywell-Jones sobre Coptotermes gestroi (Wasmann) (Isoptera: Rhinotermitidae). Arquivos do Instituto Biológico, v.78, n.4, p.565-572, 2011.

MEIKLE, W.G.; MERCADIER, G.; ROSENGAUS, R.B.; KIRK, A.A.; DEROUANÉ, F.; QUIMBY, P.C. Evaluation of an entomopathogenic fungus, Paecilomyces fumesoroseus (Wize) Brown and Smith (Deuteromycota: Hyphomyctes) obtained from Formosan subterraneamn termites (Isop., Rhinotermitidae). Journal Applied Entomology, v.129, n.6, p.315-322, 2005.

YANAGAWA, A.; YOKOHARI, F.; SHIMIZU, S. Defense mechanism of the termite, Coptotermes formosanus Shiraki, to entomopathogenic fungi. Journal of Invertebrate Pathology, v.97, n.2, p.165-170, 2008.

YANAGAWA, A.; FUJIWARA-TSUJII, N.; AKINO, T.; YOSHIMURA, T.; YANAGAWA, T. Behavioral changes in the termite, Coptotermes formosanus (Isoptera), inoculated with six fungal isolates. Journal of Invertebrate Pathology, v.107, n.2, p.100-106, 2011.

POTENZA, M.R.; ZORZENON, F.J. Cupins: Pragas em áreas urbanas. Boletim Técnico Instituto Biológico. São Paulo, 66p, 2006.

SAS INSTITUTE. SAS user's guide: Statistics, version 8.2, 6. ed. SAS Institute: Cary NC. 1999-2001.

WRIGHT, M.S.; CONNICK, W.J.JR.; JACKSON, M.A. Use of Paecilomyces spp. as pathogenic agents against subterranean termites. U.S. Patent 20030095951. 2003.

WRIGHT, M.S.; CONNICK W.J. JR; JACKSON M.A. Use of Paecilomyces spp. As pathogenic agents against subterranean termites. U.S. Patent 7390480. 2008.

WRIGHT, M.S.; CORNELIUS, M. Mortality and repellent effects of microbial pathogens on Coptotermes formosanus (Isoptera: Rhinotermitidae). BioMed Central Microbiology, v. 12, n.291, 2012 